\title{
DESIGN OF AUTOMATIC NAVIGATION CONTROL SYSTEM FOR AGRICULTURAL VEHICLE
}

\author{
Lian Zhipeng ${ }^{1}$, Zhou Zhifeng ${ }^{2}$, Chen Ning ${ }^{3}$ \\ ${ }^{1}$ Post Graduate Student, Shanghai University of Engineering Science, Shanghai, China \\ ${ }^{2}$ Professor, Shanghai University of Engineering Science, Shanghai, China \\ ${ }^{3}$ Engineer of The $R \&$ D department, Shanghai Compass Satellite Navigation Technology Co, Ltd, Shanghai, China
}

\begin{abstract}
The tractor automatic navigation technology is one of the hottest research fields of precision agriculture as well as a major means for realizing intelligent operating of agricultural vehicle in future. This paper presents a thorough research on GPS automatic navigation technology of agricultural vehicle considering that working conditions and field conditions for the tractor are complex and that there is a high requirement on the precision of the driving path of the tractor. Major contents of the research are as follows: The hydraulic control valve was selected by testing. A hydraulic control valve test platform specific to navigation was designed. The test platform can gather information about the flow and pressure of each measured hydraulic valve in a real-time manner. A navigation valve block was developed. Finally, the navigation valve block was used in electro-hydraulic transformation of model vehicle, realizing the control of electrical signal in tractor steering. A hardware platform for the automatic navigation system was built. A navigation controller based on ARM chip, the RTK-GPS positioning system and the angle sensor constitute the hardware platform of the autopilot system of the tractor. The hardware platform is the basis for realizing automatic navigation of the tractor. The navigation control algorithm was studied, pure pursuit model tracking algorithm were analyzed; the navigation decision-making control system based on the pure pursuit model tracking algorithm was designed; the kinematics model of the tractor was established. The pure tracking model has been simulated by MATLAB software, and the system has good stability and sensitivity. The experimental research on the automatic navigation system of the tractor was conducted. Based on the automatic navigation platform developed above, experiments on the control of the tractor walking straight at the flat road have been done. The results proved that the automatic navigation system has the capability of tracking the straight path of the tractor in a real-time and stable manner and meets the requirements of precision agriculture.
\end{abstract}

Keywords: Automatic Navigation, Precision Agriculture, Steering Control System, Path Tracking - ****

\section{INTRODUCTION}

The tractor automatic navigation technology is one of the hottest research fields of precision agriculture as well as a major means for realizing intelligent operating of agricultural vehicle in future. Automatic navigation technology on tractor is one of precision agriculture technology, using the leading technology ensures accurate operation, direction and distance, improving the accuracy of a job. [1-3]In addition, it can also reduce duplication of work, speed up the operation progress, reducing pilot workload.

This paper, in view of this situation, the research team designed a portable platform of agricultural vehicle, which can mimic hydraulic steering of tractors and other agricultural vehicle. A complete set of hydraulic system was installed in the vehicle. An automatic navigation control system based on BDS position system and angle sensor was developed. An algorithm for tracking path was carried out, and a calibration technique is used for calibrating this control system, by simulation and experiment, the validity of this method was testified, which explored a simple and effective method of automatic steering for agricultural vehicles.

\section{DESIGN PRINCIPLES}

In the condition of the vehicle at low speeds, there is usually no need to consider the Stability of vehicle, this paper only studied on kinematic model of the vehicle, the vehicle platform can get better control output when it uses complicated mathematical model, but there is large amount of calculation and real-time is difficult to ensure, while using a simple model may make track fail. To solve this problem, reference [4]points out, two path tracking controllers was designed respectively which used 2 degrees of freedom vehicle model and 14 degrees of freedom vehicle model, the 14 degree of freedom model could track path better, but 2 degrees of freedom model had approximate results and the time of calculation was shorter. That is to say, 2 degrees of freedom model could reflect the physical restrictions of the vehicle, so the research team used 2 degree of freedom vehicle model, which according to the symmetry of the vehicle, as Figure 1 shows, [4]through kinematic analysis, 


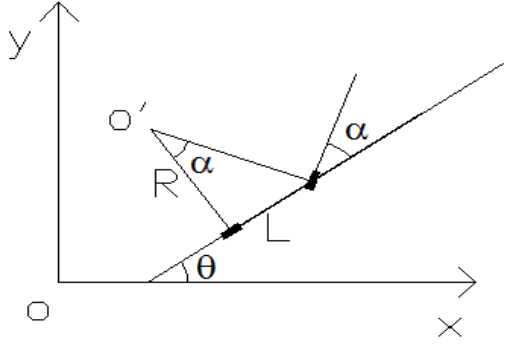

Fig -1: Simplified analysis of two-wheeled vehicle kinematic model

$$
\left\{\begin{array}{l}
x^{\prime}(t)=v(t) \cos \theta(t) \\
y^{\prime}(t)=v(t) \sin \theta(t) \\
\theta^{\prime}(t)=v(t) \tan \alpha(t) / L
\end{array}\right.
$$

$\mathrm{V}$ is vehicle speed, $\mathrm{L}$ is vehicle length, $\theta$ is heading angle, $\alpha$ is front steering angle, from equations (1) with vehicle speed, heading angle, and the front wheel steering angle, equations (2) can be derived.

$$
\left\{\begin{array}{l}
R=L / \tan \alpha \\
\theta_{k+1}=\theta_{k}+\omega \\
\Delta=R \omega \\
x_{k+1}=x_{k}+\Delta \cos \left(\theta_{k}+\omega / 2\right) \\
y_{k+1}=y_{k}+\Delta \sin \left(\theta_{k}+\omega / 2\right)
\end{array}\right.
$$

From equations(2), the vehicle trajectory equations was derived when the initial heading angle, initial coordinates and the steering angle of next time were obtained, also the coordinates of vehicle in any moment can be derived. Vehicle could trace path of the next moment when the current coordinates and the heading angle of vehicle the could be obtained by GPS, the front steering angle can be obtained by angle sensor. This is the basic principle of navigation control.

\section{THE OVERALL STRUCTURE}

This automatic navigation control system could be divided into GNSS satellite positioning system, data communication system, as well as hydraulic steering system.

Navigation system is critical for navigation control system, which mainly included ARM Micro-controller, GNSS Navigation receiver, angle sensor and other components. The system consisted of M300C-U base station, UDL300 station and M600U satellite receiver with double antennas. M300C-U modulation as a satellite base station and could radio data signal, mobile station UDL300 demodulated signals of base station radio, with GPS data provided by M600U dual-antenna receiver, which obtained navigation coordinates and direction information by RTK technology. The ARM controller received the navigation information, processed to obtain the control signal, transmitted it to the hydraulic actuator. MCGJ100A38CHF angle sensor was installed on the left front wheel of vehicle, which stored data of front wheel steering angle, and fed back to ARM controller. The hydraulic subsystem received the control signal to steer. Architecture diagram is shown in Figure 2:

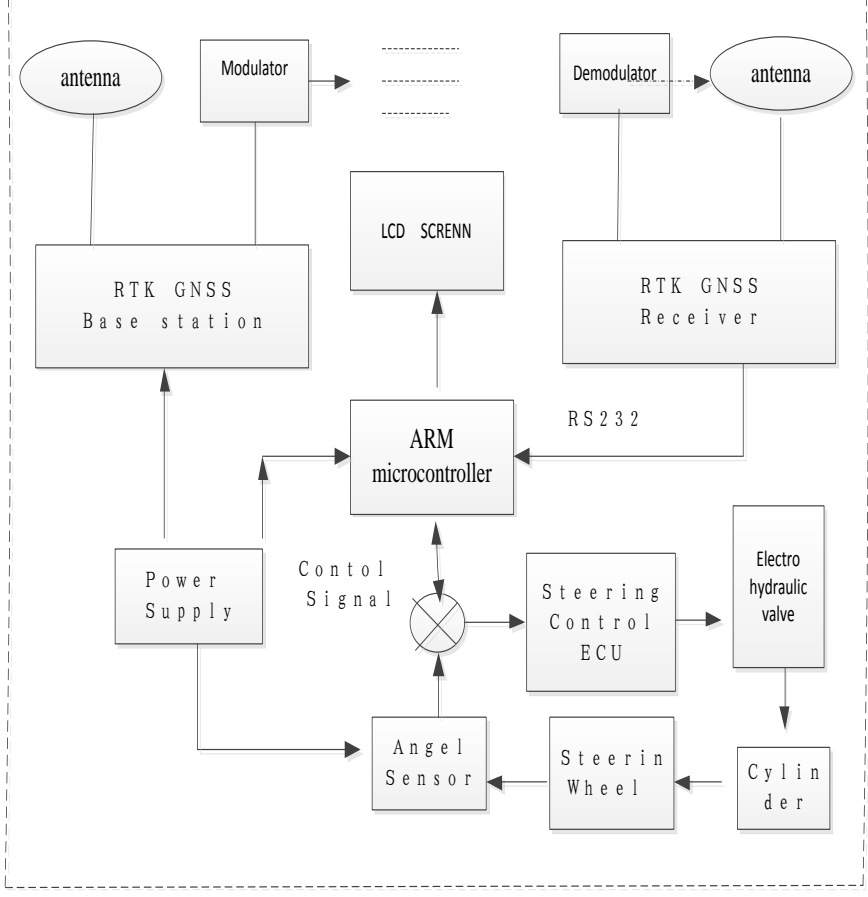

Fig -1: Name of the figure

\section{THE HARDWARE OF SYSTEM}

\subsection{Electro-Hydraulic Steering System}

Paragraph Automatic steering system was important for automatic driving vehicles, which determined whether agricultural vehicles could correctly and accurately work or not. The basic working principle of automatic steering technology was the automatic steering control system constantly adjusted front-wheel steering angle to track predefined path.

The research team studied electro-hydraulic steering system commonly used on tractor, and designed a portable platform of agricultural vehicle to mimic hydraulic steering of tractors. The design of the hydraulic steering mechanism is shown in Figure 3, including cylinder, oil tank, oil pump, brushless DC motor, pressure gauge, flow limiting valve, tubing and electromagnetic proportional valve. 4 liter fuel tank with pump mechanism and battery supply was installed behind the platform. Hydraulic valves and tubing could be installed under the model platform vehicle, so it could ensure the best visual impression of the vehicle. The proportional valve is the role to control the steering speed by flow control, electromagnetic reversing valves is for the completion of the switching direction, then driving the front wheels turn left or right. [6]In order to accurately respond to control output, the research team calibrated the hydraulic steering angle. 


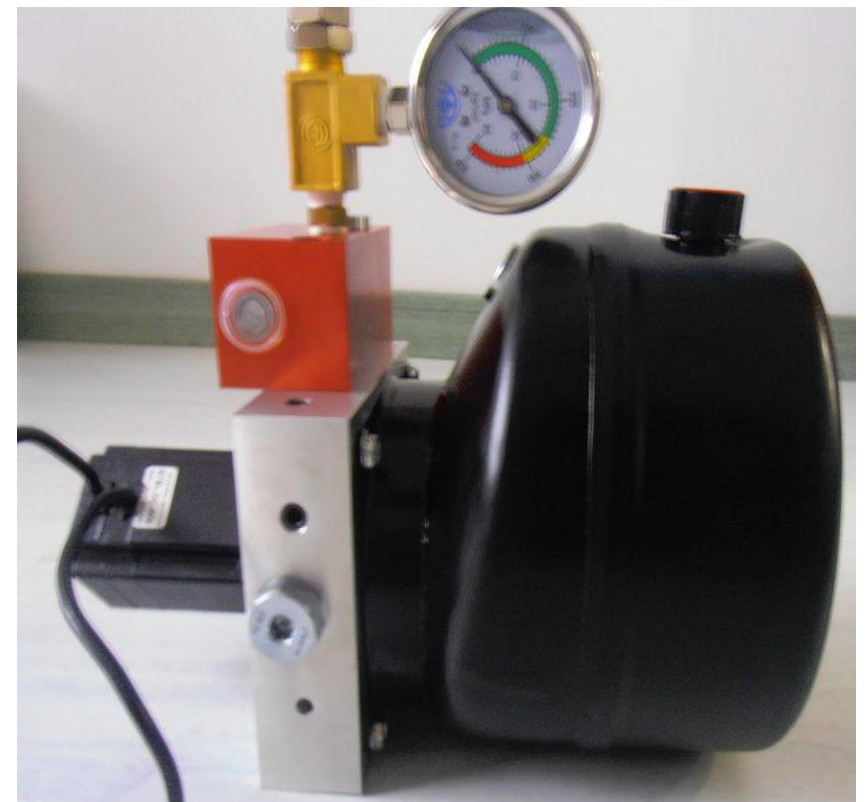

Fig -3: Fuel tank pumps and the installation location photo

The signal of output was obtained by the SPI interface of ARM microcontroller, the output signal is $0-2.5 \mathrm{~V}$ analog voltage, which is converted to $-10 \mathrm{~V} \sim+10 \mathrm{~V}$ analog voltage through the OPA188AIDGKT amplifier, then the control value of three-potential four-way valve can be obtained through the DAC8551_DGK_8 converter.

The correlation between $-10 \mathrm{~V} \sim+10 \mathrm{~V}$ analog voltage and the actual steering angle is determined by experiments, in view of different structure of the hydraulic cylinders, the steering angle of the same output voltage is different on the left turning and right turning. Left steering angle and output voltage correlation is shown in Table 1, right steering angle and output voltage correlation is shown in table 2 .

Table -1: Left steering angle and output voltage relationship

\begin{tabular}{llllll}
\hline $\begin{array}{l}\text { Out- } \\
\text { put } \\
(\mathrm{V})\end{array}$ & $\begin{array}{l}\text { left } \\
\text { angle } \\
\left({ }^{\circ}\right)\end{array}$ & $\begin{array}{l}\text { Out- } \\
\text { put } \\
(\mathrm{V})\end{array}$ & $\begin{array}{l}\text { left } \\
\text { angle } \\
\left(^{\circ}\right)\end{array}$ & $\begin{array}{l}\text { Out- } \\
\text { put } \\
(\mathrm{V})\end{array}$ & $\begin{array}{l}\text { left } \\
\text { angle } \\
\left({ }^{\circ}\right)\end{array}$ \\
\hline 2 & 3.0 & 5 & 6.6 & 8 & 10.2 \\
2.5 & 3.6 & 5.5 & 7.9 & 8.5 & 10.1 \\
3 & 4.1 & 6 & 8.0 & 9 & 10.4 \\
3.5 & 4.2 & 6.5 & 8.3 & 9.5 & 11.3 \\
4 & 5.7 & 7 & 8.4 & 10 & 12.8 \\
4.5 & 6.2 & 7.5 & 9.3 & & \\
& & & & & \\
\hline
\end{tabular}

Table -2: Right steering angle and output voltage relationship

\begin{tabular}{llllll}
\hline $\begin{array}{l}\text { Out- } \\
\text { put } \\
(\mathrm{V})\end{array}$ & $\begin{array}{l}\text { left } \\
\text { angle } \\
\left({ }^{\circ}\right)\end{array}$ & $\begin{array}{l}\text { Out- } \\
\text { put } \\
(\mathrm{V})\end{array}$ & $\begin{array}{l}\text { left } \\
\text { angle } \\
\left(^{\circ}\right)\end{array}$ & $\begin{array}{l}\text { Out- } \\
\text { put } \\
(\mathrm{V})\end{array}$ & $\begin{array}{l}\text { left } \\
\text { angle } \\
\left({ }^{\circ}\right)\end{array}$ \\
\hline 2 & 1.1 & 5 & 4.7 & 8 & 8.6 \\
& & & & & \\
2.5 & 1.6 & 5.5 & 5.6 & 8.5 & 8.1 \\
3 & 1.9 & 6 & 6.2 & 9 & 8.8 \\
3.5 & 2.8 & 6.5 & 5.9 & 9.5 & 10.3 \\
4 & 3.8 & 7 & 6.7 & 10 & 10.8 \\
4.5 & 4.6 & 7.5 & 7.6 & & \\
\hline
\end{tabular}

\subsection{Dual- Directional-Antenna Measurements}

Location information and direction information is two key parameters for automatic navigation, Location coordinates information can be obtained directly from the satellite receiving system, while the direction information is obtained by the measurement of double antenna.

Two GNSS antenna were installed in the front and rear of the vehicle for attitude measurement. Once two antennas were installed, the distance between the two antennas is constant, which can be accurately respectively measured by GNSS. [7]it is shown in the figure 4, coordinates of Antenna 2 is $\left(L_{2}, 0,0\right)$, coordinates of Antenna 1 is $(0,0,0)$, and $L 2$ is the distance between the two antennas. Heading and roll angle can be derived directly:

$$
\begin{gathered}
\psi=\arctan \left(\frac{\mathrm{y}_{2}^{L}}{x_{2}^{L}}\right) \\
\theta=\arctan \left(\frac{Z_{2}^{L}}{\sqrt{\left(x_{2}^{L}\right)^{2}+\left(\mathrm{y}_{2}^{L}\right)^{2}}}\right)
\end{gathered}
$$

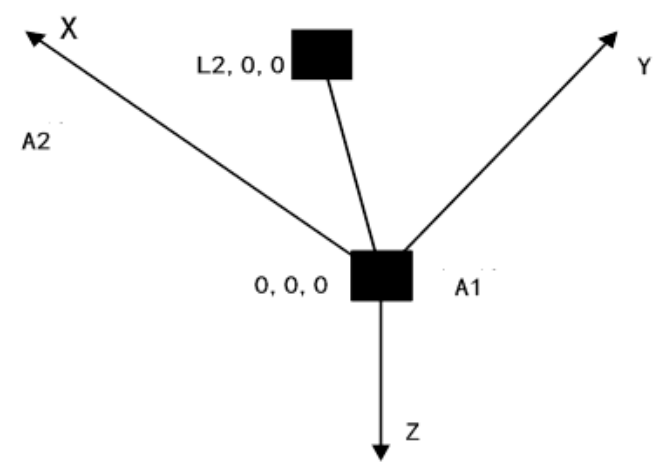

Fig -4: Dual antenna heading measurement chart diagram

Antenna $A 1$ compared with the antenna $A 2$ in the threedimensional location of geodetic coordinate system can be accurately measured, coordinates of two antennas based on the geodetic coordinate system are transformed into coordinate system which based on the local level coordinate system that $A l$ as the origin, then the coordinate data is substituted into equation (3) (4) to obtain heading angle and roll angle. 


\subsection{Front Wheel Angle Measurement}

MCGJ100A38CHF module is used as angle sensor. The shaft of angle sensor is installed together with the steering arm of front wheel through a three-link, mechanism graph is shown in Figure 5, The shaft of angel sensor will rotate with the rotation of the front wheel when the front wheel of the vehicle steers.

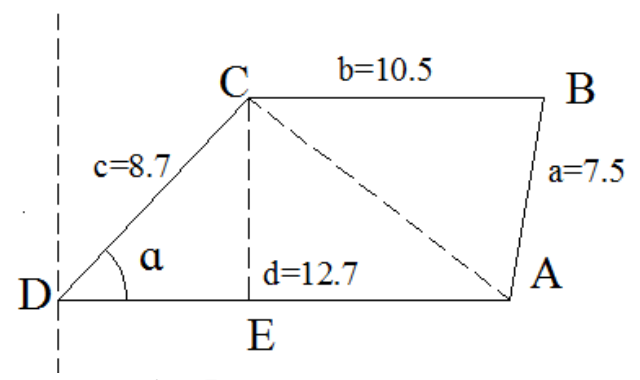

Fig -5: Three Linkage map

$D$ point is the center point of shaft, $A$ point is the connection point between linkage $\mathrm{AB}$ and steering arm of front wheel. $a=7.5 \mathrm{~cm}, b=10.5 \mathrm{~cm}, d=12.7 \mathrm{~cm}, c=8.7 \mathrm{~cm} . \alpha$ is steering angle of the angle sensors, $\beta$ is the front wheel steering angle.

Because the range of the front wheel steering angle is $0 \sim 40^{\circ}$ , the range of the angle sensor is from the $18^{\circ}$ to $98^{\circ}$. The mapping model of correlation between front-wheel steering angle and angle sensor is established. The data can be drawn, which is shown in Figure.

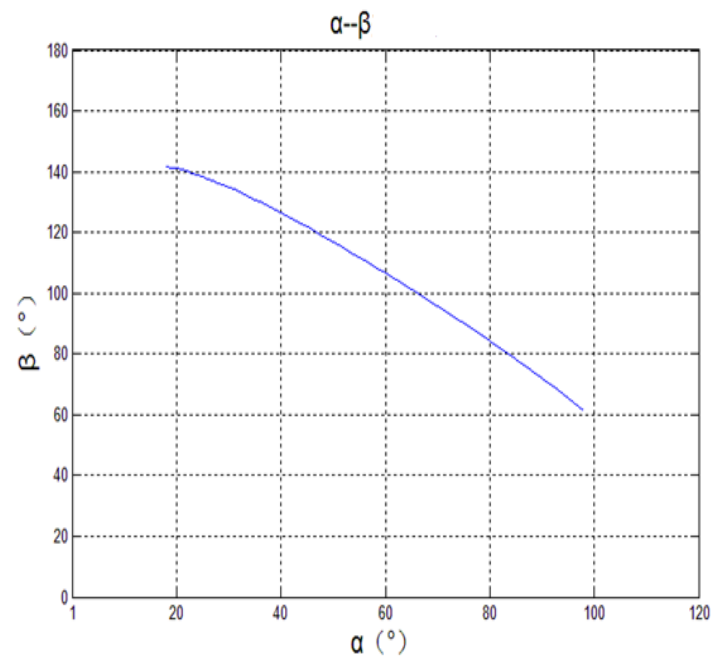

Fig -6: Steering shaft angle sensor and the front wheel steering angle diagram

\section{PATH TRACKING ALGORITHM}

According to the characteristics of the kinematics of agricultural vehicle model and the pure pursuit model, the research team pointed out that the tracking error comes mainly from the heading angle and the steering angle. The research team proposed an improved algorithm of pure pursuit model.
As it shown in Figure 7, according to pure pursuit algorithm, the vehicle drives with expected steering angle, in the first control cycle, vehicle arrives at the $P_{1}\left(x_{1}, y_{1}\right)$, steering angle and roll angle are not 0 , vehicle deviates from the expected line. The roll angle and the steering angle will be 0 by adjusting the steering angle within the next two control cycle. After the second cycle, the vehicle arrives at $P_{2}\left(x_{2}\right.$, $y_{2}$ ), the expected steering angle is 0 , after the third cycles, the vehicle arrives at $P\left(x^{\prime}, y^{\prime}\right)$, the expected heading angle and expected steering of the vehicle are both 0 , steady state is achieved.

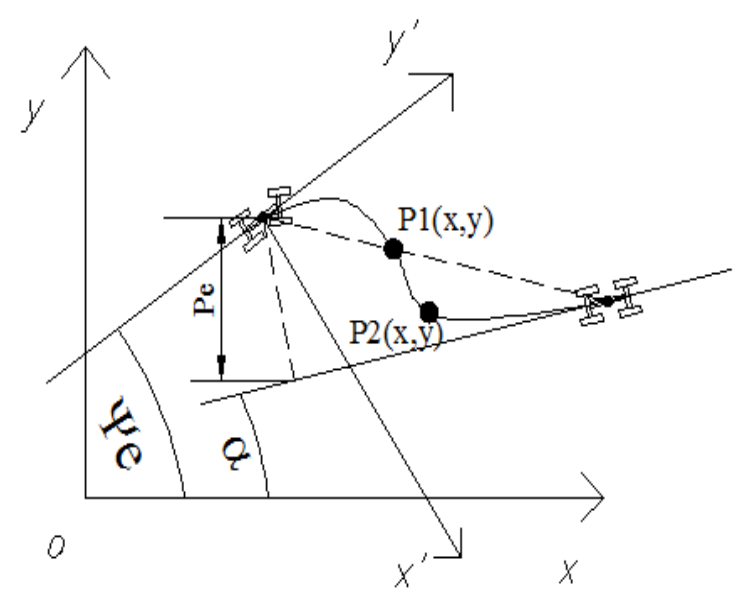

Fig -7: Vehicle path tracking map of improved pure pursuit algorithm

[8]The formula for improved algorithm of pure pursuit model is

$$
\left\{\begin{array}{l}
\theta_{1}=\arctan \left[\frac{2 H\left(P e \cos \psi_{e}-\sqrt{L^{2}-P e^{2}} \sin \left(\psi_{e}\right)\right.}{L^{2}}\right] \\
\theta_{2}=\frac{H \cdot \psi_{e}}{V \cdot T}-\theta_{0}
\end{array}\right.
$$

The $\psi_{e}$ is the angle between current heading angel and the expected path, $P_{e}$ is lateral tracking error between vehicle and expected path, $\mathrm{H}$ is length of the vehicle. $\theta_{0}$ is the actual steering angle before correction, $\theta_{l}$ is the steering angle after the first correction of the improved algorithm of pure pursuit model, $\theta_{2}$ is the steering angle after the second correction.

The simulation of improved algorithm of pure pursuit model is carried out in MATLAB/SIMULINK environment. Simulation conditions: $\mathrm{AB}$ line, starting point is $A(6,8)$, terminal point is $B(250,200)$, and the $\mathrm{AB}$ line starts from the same starting point of vehicle, the location error is $0.4 \mathrm{~m}$, heading angle measurement error is $0.5^{\circ}$, the steering angle error is $0.6^{\circ}$, the speed error is in $0.2 \mathrm{~m} / \mathrm{s}$. The maximum steering angle is $20^{\circ}$, the control cycle $T=1 \mathrm{~s}$, the length of the vehicle is $2 \mathrm{~m}$. When $V=2 \mathrm{~m} / \mathrm{s}, L=4 \mathrm{~m}$, simulation of tracking error of improved pure pursuit algorithm is shown in Figure 9 : 


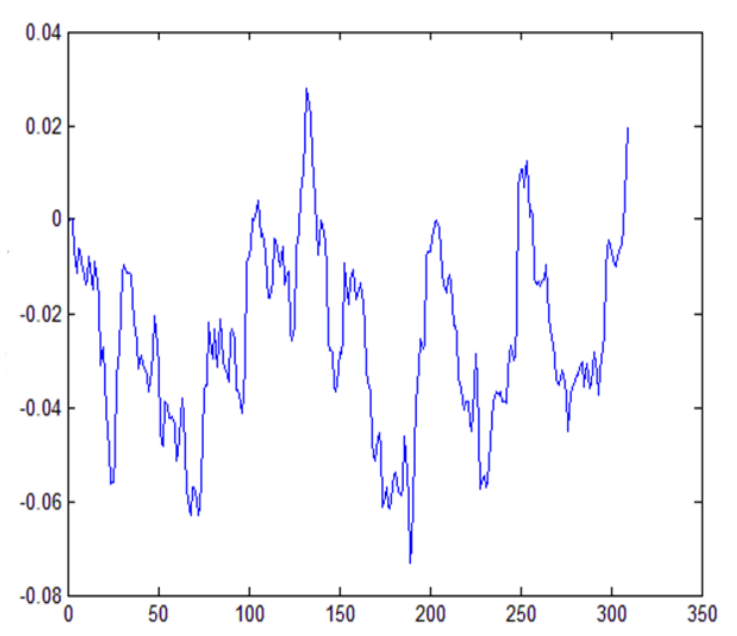

Fig -8: Tracking error chart of improved pure pursuit algorithm

Simulation result: average error is $0.0233 \mathrm{~m}$, maximum error of the car is $0.0853 \mathrm{~m}$. The result shows that the improved algorithm in long distance and large original error tracking is effective.

\section{AUTOMATIC NAVIGATION AND DRIVING}

\section{TESTS}

Paragraph The Experiment vehicle is shown in Figure 9, the vehicle is equipped with BDS/GPS receiver, dual antenna, hydraulic steering control system, ARM control board, angle sensor, which consist experiment platform. The location of experiment was in the square in front of library in the campus.

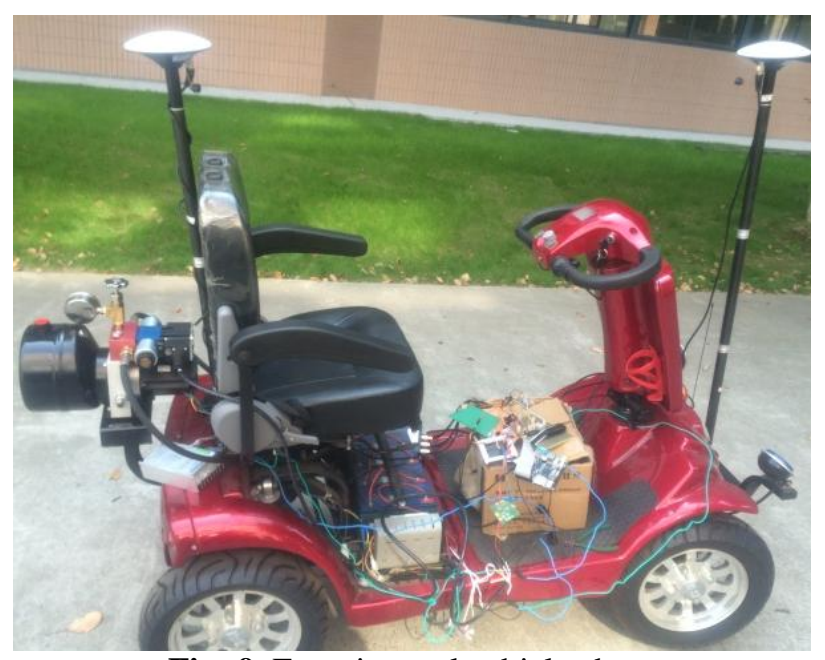

Fig -9: Experimental vehicle photo

The research team tested the automatic driving agricultural vehicle in the campus, set A point then moved the vehicle to B point away about $350 \mathrm{~m}$, Click the Start Button on the screen in the vehicle, vehicle was moving, the controller will control the front wheel steering agricultural vehicle in realtime, the speed of the vehicle was $1 \mathrm{~m} / \mathrm{s}$, the visibility distance was $3 \mathrm{~m}$ when it tracked path. Test results are shown in Figure 9, experiments were conducted several times, experiment data was recorded ten times, statistical data is shown in table 3 .

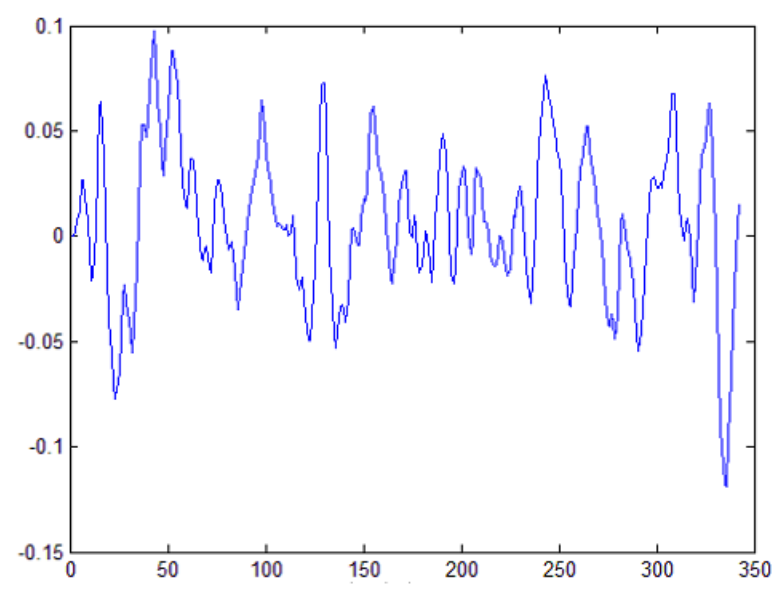

Fig -10: Path tracking map

Table -3: Path tracing lateral error statistics

\begin{tabular}{lll}
\hline TEST.NO & $\begin{array}{l}\text { Average } \\
\text { Error }(\mathrm{m})\end{array}$ & $\begin{array}{l}\text { The maximum } \\
\text { Error }(\mathrm{m})\end{array}$ \\
\hline 1 & 0.0586 & 0.1186 \\
2 & 0.0556 & 0.1023 \\
3 & 0.0546 & 0.1078 \\
4 & 0.0567 & 0.1024 \\
5 & 0.0578 & 0.1036 \\
6 & 0.0607 & 0.1170 \\
7 & 0.0592 & 0.1035 \\
8 & 0.0593 & 0.1062 \\
9 & 0.0513 & 0.1105 \\
10 & 0.0589 & 0.1199 \\
\hline
\end{tabular}

The experimental results are as follows: When tractor walks on the flat road automatically at a speed of $1 \mathrm{~m} / \mathrm{s}$, the maximum lateral deviation is $0.1199 \mathrm{~m}$, the average error is $0.0574 \mathrm{~m}$. The experimental results indicated that the performance of controller is good, and it can be used in the steering control system

\section{CONSLUSION}

To study the automatic guidance control of precision agricultural vehicle, a navigation control system was developed which integrated with computer technology, sensor technology, GPS technology and communication technology. The navigation control system could control the platform of the agriculture vehicle to trace the predefined trajectory using GNSS and angel sensor. The navigation control algorithm was studied, pure pursuit model tracking algorithm were analyzed; the navigation decision-making control system based on the pure pursuit model tracking algorithm was designed; the kinematics model of the tractor was established. Simulation results based on simplified vehicle model verified the validity of the algorithms. to test the performance of the developed electric hydraulic power steering system, the part of the transformation system was 
simulated with MATLAB, and it took the outdoor experiments. The result showed that the electric hydraulic power steering system had the advantages of large torque, fast response speed and high steering precision. automatic navigation system has the capability of tracking the straight path of the tractor in a real-time and stable manner and meets the requirements of precision agriculture.

\section{ACKNOWLEDGEMENTS}

The author would like to thank Professor Zhou Zhifeng who helped and supported with this work. This project was sponsored by Shanghai University of Engineering Science Innovation Fund for Graduate Students (No.14KY0131)

\section{REFERENCES}

[1]. Huang Peichen, Luo Xiwen, Zhang Zhigang. Control method of headland turning based on improved pure pursuit model for agricultural machine [J].Computer Engineering and Applications, 2010,46(21):216-219.

[2]. Zhang Meina, Lin Xiangze, Ding Yongqian, et al. Design of path following controllers based on performance index for agricultural vehicl [J].Transactions of the Chinese Society of Agricultural Engineering,2012,28(9):40-46.

[3]. Zhou Jianjun, Zhang Man, Wang Maohua, et al. Path tracking for agricultural vehicle based on fuzzy control [J]. Transactions of the Chinese Society for Agricultural Machinery, 2009, 40(4):151-156.

[4]. Zhang Zhigang, Luo Xiwen, Zhao Zuoxi, etal. Trajectory tracking control method based on Kalman filter and pure pursuit model for agricultural vehicle[J] .Transactions of the Chinese Society for Agricultural Machinery, 2009,40(Supp.):6-12.

[5]. Lian Shijiang, Chen Jun, Jia Haizheng, et a1. Automatic turning control system of tractor based on PID control[J]. Journal of Agricultural Mechanization Research,2009, 6(6): 211--213.

[6]. Li Jin, Chen Wuwei. Navigation of vision-guided intelligent vehicle based on adaptive navigation parameters [J].Transactions of the Chinese Society for Agricultural Machinery,2012,43(6):19 -24.

[7]. Liu J, Jayakumar P, Overholt J L, et al. The Role of Model Fidelity in Model Predictive Control Based Hazard Avoidance in Unmanned Ground Vehicles Using LIDAR Sensors[C]. Proceedings of the ASME Dynamic Systems and Control Conference,2013:1-10.

[8]. Li Taochang, $\mathrm{Hu}$ Jingtao, Gao Lei1, et al. Agricultural machine path tracking method based on fuzzy adaptive pure pursuit model[J].Transactions of the Chinese Society for Agricultural Machinery, 2013, 44(1):205-210.

\section{BIOGRAPHIES}

Lian Zhipeng, Post Graduate Student, Post Graduate Student, Shanghai University of Engineering Science, Shanghai, China

Zhou Zhifeng, Professor, Shanghai University of Engineering Science, Shanghai, China
Chen Ning, Engineer of The R \& D department, Shanghai Compass Satellite Navigation Technology Co, Ltd, Shanghai, China 